\title{
Radiative heat transfer between nanostructures
}

\author{
A. I. Volokitin ${ }^{1,2}$ and B. N. J. Persson ${ }^{1}$ \\ ${ }^{1}$ Institut für Festkörperforschung, Forschungszentrum Jülich, D-52425, Germany \\ ${ }^{2}$ Samara State Technical University, 443010 Samara, Russia \\ (Received 14 July 2000; revised manuscript received 25 September 2000; published 16 April 2001)
}

\begin{abstract}
We use a general theory of the fluctuating electromagnetic field and a generalized Kirchhoff's law (Ref. 8) to calculate the heat transfer between macroscopic and nanoscale bodies of arbitrary shape, dispersive, and absorptive dielectric properties. We study the heat transfer between: (a) two parallel semi-infinite bodies, (b) a semi-infinite body and a spherical body, and (c) two spherical bodies. We consider the dependence of the heat transfer on the temperature $T$, the shape and the separation $d$, and discuss the role of nonlocal and retardation effects. We find that for low-resistivity material the heat transfer is dominated by retardation effects even for the very short separations.
\end{abstract}

DOI: 10.1103/PhysRevB.63.205404

PACS number(s): $65.80 .+\mathrm{n}$

\section{INTRODUCTION}

It is well known that for bodies separated by $d \gg d_{W}$ $\sim c \hbar / k_{B} T$ the radiative heat transfer between them is described by the Stefan-Bolzman law:

$$
J=\frac{\pi^{2} k_{B}^{4}}{60 \hbar^{3} c^{2}}\left(T_{1}^{4}-T_{2}^{4}\right),
$$

where $T_{1}$ and $T_{2}$ are the temperatures of solid 1 and 2, respectively. In this limiting case the heat transfer is connected with traveling electromagnetic waves radiated by the bodies, and does not depend on the separation $d$. For $d<d_{W}$, the heat transfer increases by many order of magnitude, which can be explained by the existence of evanescent electromagnetic field that decay exponentially into the vacuum. At the present time there is an increasing number of investigations of heat transfer due to evanescent waves in connection with scanning tunneling microscopy and scanning thermal microscopy (STM) under ultrahigh vacuum conditions. ${ }^{1-4}$ STM can be used for local heating of the surface, resulting in local desorption or decomposition of molecular species, and this offers further possibilities for the STM to control local chemistry on a surface.

A general formalism for evaluating the heat transfer between macroscopic bodies was proposed some years ago by Polder and Van Hove. ${ }^{1}$ Their theory is based on the general theory of the fluctuating electromagnetic field developed by Rytov $^{5}$ and applied by Lifshitz ${ }^{6}$ for studying the conservative part, and by Volokitin and Persson ${ }^{7}$ for studying the dissipative part of the van der Waals interaction. The formalism of Polder and Van Hove can be significantly simplified using a generalized Kirchhoff's law. ${ }^{2,8}$ In this approach, the calculation of the correlation functions for the fluctuating electromagnetic field is reduced to finding the electromagnetic field created by a point dipole outside the bodies. The formalism of Polder and Van Hove requires the determination of the electromagnetic field for all space and for all position of a point dipole, and requires the integration of the product of the component of the electric and magnetic field over the volumes of two bodies. In the present paper we use a simpler formalism, which is originally due to Levin and Rytov. ${ }^{8}$ This formalism requires only the evaluation of a surface integral over one of the bodies and is simplified further in the nonretarded limit (small distances between bodies), where the calculation of the heat transfer is reduced to the problem of finding the electrostatic potential due to a point charge. We apply the formalism to the calculation of the heat transfer between: (a) two semi-infinite bodies, (b) a semi-infinite body and a spherical particle, and (c) two spherical particles. Problem (a) was considered by Polder and Van Hove, ${ }^{1}$ Levin, Polevoy, and Rytov, ${ }^{2}$ and more recently by Pendry. ${ }^{3}$ In comparison with other treatments, we study in detail the nonlocal and retardation effects. A striking result we find is that for low-resistivity metals retardation effects become crucial and in fact dominate the heat transfer between bodies. The problem (b) was recently studied by Pendry in a different formalism. ${ }^{3}$ We shall point out the differences between our results and those obtained by Pendry, wherever appropriate.

\section{FORMALISM}

Following Polder and Van Hove, ${ }^{1}$ to calculate the fluctuating electromagnetic field we use the general theory of Rytov (see Refs. 5,8). This method is based on the introduction of a fluctuating current density in the Maxwell equations (just as, for example, the introduction of a "random" force in the theory of Brownian motion of a particle). For a monochromatic field [time factor $\exp (-i \omega t)$ ] in a dielectric, nonmagnetic medium, these equations are

$$
\begin{gathered}
\boldsymbol{\nabla} \times \mathbf{E}=i \frac{\omega}{c} \mathbf{B}, \\
\boldsymbol{\nabla} \times \mathbf{H}=-i \frac{\omega}{c} \mathbf{D}+\frac{4 \pi}{c} \mathbf{j}^{f},
\end{gathered}
$$

where, according to Rytov, we have introduced a fluctuating current density $\mathbf{j}^{f}$ associated with thermal and quantum fluctuations. E, D, $\mathbf{H}$, and $\mathbf{B}$ are the electric and the electricdisplacement field, and the magnetic and the magneticinduction fields, respectively. For non-magnetic media B $=\mathbf{H}$ and $\mathbf{D}=\varepsilon \mathbf{E}$, where $\varepsilon$ is the dielectric constant of the 
surrounded media. Accordingly to Rytov, the average value of the product of components of $\mathbf{j}^{f}$ for the local optic case is given by formula

$$
\begin{gathered}
\left\langle j_{i}^{f}(\mathbf{r}, \omega) j_{k}^{f *}\left(\mathbf{r}^{\prime}, \omega^{\prime}\right)\right\rangle=\left\langle j_{i}^{f}(\mathbf{r}) j_{k}^{f^{*}}\left(\mathbf{r}^{\prime}\right)\right\rangle_{\omega} \delta\left(\omega-\omega^{\prime}\right), \\
\left\langle j_{i}^{f}(\mathbf{r}) j_{k}^{*}\left(\mathbf{r}^{\prime}\right)\right\rangle_{\omega}=A(T, \omega) \omega^{2} \operatorname{Im} \varepsilon(\omega) \delta\left(\mathbf{r}-\mathbf{r}^{\prime}\right) \delta_{i k}, \\
A(T, \omega)=\frac{\hbar}{(2 \pi)^{2}}\left(\frac{1}{2}+n(\omega)\right), \\
n(\omega)=\frac{1}{e^{\hbar \omega / k_{B} T}-1},
\end{gathered}
$$

and for nonlocal optic

$$
\left\langle j_{i}^{f}(\mathbf{r}) j_{k}^{f^{*}}\left(\mathbf{r}^{\prime}\right)\right\rangle_{\omega}=A(T, \omega) \omega^{2} \operatorname{Im} \varepsilon_{i k}\left(\mathbf{r}, \mathbf{r}^{\prime}, \omega\right),
$$

where $\varepsilon_{i k}\left(\mathbf{r}, \mathbf{r}^{\prime}, \omega\right)$ is a nonlocal dielectric constant. To calculate the correlation functions for the fluctuating electromagnetic field, we use the theory based on the generalized Kirchhoff's law. ${ }^{8}$ For simplicity, in the derivation we will assume local optics. However, the same derivation is valid also for the nonlocal optics case, and the final result is the same in the sense that in both cases the problem of the heat transfer between two bodies is reduced to the problem of finding the electromagnetic field outside the bodies. Compared to Polder and Van Hove, this treatment includes nonlocal effects, such as the anomalous skin effect.

In order to calculate the radiative energy transfer between the bodies, we need the ensemble average of the Poyting vector

$$
\langle\mathbf{S}(\mathbf{r})\rangle_{\omega}=(c / 8 \pi)\left\langle\mathbf{E}(\mathbf{r}) \times \mathbf{B}^{*}(\mathbf{r})\right\rangle_{\omega}+\text { c.c. },
$$

at suitable point $\mathbf{r}$. From Maxwell equations it follows that the electric field produced by random current density $\mathbf{j}^{f}$, is given by

$$
E_{i}(\mathbf{r})=\int d \mathbf{r}^{\prime} D_{i k}\left(\mathbf{r}, \mathbf{r}^{\prime}, \omega\right) j_{k}^{f}\left(\mathbf{r}^{\prime}\right),
$$

where function $D_{i k}\left(\mathbf{r}, \mathbf{r}^{\prime}, \omega\right)$ obeys the equations

$$
\begin{aligned}
& {\left[\nabla_{i} \nabla_{k}-\delta_{i k}\left\{\nabla^{2}-(\omega / c)^{2}\right\} \varepsilon(\mathbf{r})\right] D_{k j}\left(\mathbf{r}, \mathbf{r}^{\prime}, \omega\right)} \\
& \quad=\left(4 \pi \omega i / c^{2}\right) \delta_{i j} \delta\left(\mathbf{r}-\mathbf{r}^{\prime}\right), \\
& {\left[\nabla_{j}^{\prime} \nabla_{k}^{\prime}-\delta_{j k}\left\{\nabla^{\prime 2}-(\omega / c)^{2}\right\} \varepsilon\left(\mathbf{r}^{\prime}\right)\right] D_{i k}\left(\mathbf{r}, \mathbf{r}^{\prime}, \omega\right)} \\
& \quad=\left(4 \pi \omega i / c^{2}\right) \delta_{i j} \delta\left(\mathbf{r}-\mathbf{r}^{\prime}\right) .
\end{aligned}
$$

The functions $D_{i k}\left(\mathbf{r}, \mathbf{r}^{\prime}, \omega\right)$ have the following symmetry properties: ${ }^{9}$

$$
D_{i k}\left(\mathbf{r}, \mathbf{r}^{\prime}, \omega\right)=D_{k i}\left(\mathbf{r}^{\prime}, \mathbf{r}, \omega\right) .
$$

The Poynting vector can be expressed trough the average products of the components of the electric field. Using Eqs. (10) and (11) we get

$$
\begin{aligned}
\left\langle E_{i}(\mathbf{r}) E_{j}^{*}\left(\mathbf{r}^{\prime}\right)\right\rangle_{\omega} \\
=\int d \mathbf{r}^{\prime \prime} A\left(\mathbf{r}^{\prime \prime}\right) \omega^{2} \operatorname{Im} \varepsilon\left(\mathbf{r}^{\prime}\right) D_{i k}\left(\mathbf{r}, \mathbf{r}^{\prime}\right) D_{j k}^{*}\left(\mathbf{r}^{\prime}, \mathbf{r}^{\prime \prime}\right) \\
=\frac{\left[A\left(T_{1}\right)-A\left(T_{2}\right)\right] c^{2}}{2 i} \int d S_{11}^{\prime \prime}\left\{D_{i k}\left(\mathbf{r}, \mathbf{r}^{\prime \prime}\right)\right. \\
\quad \times\left[\nabla_{l}^{\prime \prime} D_{j k}^{*}\left(\mathbf{r}^{\prime}, \mathbf{r}^{\prime \prime}\right)-\nabla_{k}^{\prime \prime} D_{j l}^{*}\left(\mathbf{r}^{\prime}, \mathbf{r}^{\prime \prime}\right)\right]-D_{j k}^{*}\left(\mathbf{r}^{\prime}, \mathbf{r}^{\prime \prime}\right) \\
\left.\quad \times\left[\nabla_{l}^{\prime \prime} D_{i k}\left(\mathbf{r}, \mathbf{r}^{\prime}\right)-\nabla_{k}^{\prime \prime} D_{j i}\left(\mathbf{r}, \mathbf{r}^{\prime}\right)\right]\right\} \\
\quad-4 \pi \omega A\left(T_{2}\right) \operatorname{Re} D_{i j}\left(\mathbf{r}, \mathbf{r}^{\prime}\right),
\end{aligned}
$$

where we transformed the volume integral over bodies 1 and 2 to a surface integral over body 1 . Assume that the two points $\mathbf{r}$ and $\mathbf{r}^{\prime}$ lie outside the bodies. Using that for $\mathbf{r} \neq \mathbf{r}^{\prime}$

$D_{i k}\left(\mathbf{r}, \mathbf{r}^{\prime}\right) \nabla_{k}^{\prime \prime} D_{j l}^{*}\left(\mathbf{r}^{\prime}, \mathbf{r}^{\prime \prime}\right)=\nabla_{k}^{\prime \prime}\left[D_{i k}\left(\mathbf{r}, \mathbf{r}^{\prime}\right) D_{j l}^{*}\left(\mathbf{r}^{\prime}, \mathbf{r}^{\prime \prime}\right)\right]$,

and performing surface integral in Eq. (13) gives

$$
\begin{aligned}
\left\langle E_{i}(\mathbf{r}) E_{j}^{*}\left(\mathbf{r}^{\prime}\right)\right\rangle_{\omega} & \\
= & \frac{\left[A\left(T_{1}\right)-A\left(T_{2}\right)\right] c^{2}}{2 i} \int d \mathbf{S}_{1}^{\prime \prime} \cdot\left\{D_{i k}\left(\mathbf{r}, \mathbf{r}^{\prime \prime}\right) \nabla^{\prime \prime} D_{j k}^{*}\left(\mathbf{r}^{\prime}, \mathbf{r}^{\prime \prime}\right)\right. \\
& \left.-D_{j k}^{*}\left(\mathbf{r}^{\prime}, \mathbf{r}^{\prime \prime}\right) \nabla^{\prime \prime} D_{i k}\left(\mathbf{r}, \mathbf{r}^{\prime \prime}\right)\right\}-4 \pi \omega A\left(T_{2}\right) \operatorname{Re} D_{i j}\left(\mathbf{r}, \mathbf{r}^{\prime}\right) .
\end{aligned}
$$

Using the Maxwell Eq. (2) we can write the Poyting vector as

$$
\begin{aligned}
\langle\mathbf{S}\rangle_{\omega}= & \frac{i c^{2}}{8 \pi \omega}\left\{\boldsymbol{\nabla}^{\prime}\left\langle\mathbf{E}(\mathbf{r}) \cdot \mathbf{E}^{\prime}\left(\mathbf{r}^{\prime}\right)\right\rangle\right. \\
& \left.-\left\langle\left(\mathbf{E}(\mathbf{r}) \cdot \nabla^{\prime}\right) \mathbf{E}^{*}\left(\mathbf{r}^{\prime}\right)\right\rangle-\text { c.c. }\right\}_{\mathbf{r}=\mathbf{r}^{\prime}}
\end{aligned}
$$

In the nonretarded limit the formalism can be simplified. In this case, the electric field can be written as the gradient of an electrostatic potential, $\mathbf{E}(\mathbf{r})=-\nabla \phi(\mathbf{r})$. Thus, the total Poynting vector becomes

$$
\begin{aligned}
\left(S_{\text {total }}\right)_{\omega} & =\frac{c}{8 \pi} \int d \mathbf{S} \cdot\left\{\left\langle\left[\mathbf{E} \times \mathbf{B}^{*}\right]\right\rangle_{\omega}+\text { c.c. }\right\} \\
& =\frac{i \omega}{8 \pi} \int d \mathbf{S} \cdot \nabla^{\prime}\left(\left\langle\phi(\mathbf{r}) \phi^{*}\left(\mathbf{r}^{\prime}\right)\right\rangle_{\omega}-\text { c.c. }\right)_{\mathbf{r}=\mathbf{r}^{\prime}}
\end{aligned}
$$

In the same approximation we can write

$$
D_{i k}\left(\mathbf{r}, \mathbf{r}^{\prime}\right)=-\frac{i}{\omega} \nabla_{i} \nabla_{k}^{\prime} D\left(\mathbf{r}, \mathbf{r}^{\prime}\right)
$$

where the function $D\left(\mathbf{r}, \mathbf{r}^{\prime}\right)$ obeys the Poisson's equation

$$
\Delta D\left(\mathbf{r}, \mathbf{r}^{\prime}\right)=-4 \pi \delta\left(\mathbf{r}-\mathbf{r}^{\prime}\right) .
$$

Using the identities 


$$
\begin{aligned}
D_{i k}\left(\mathbf{r}, \mathbf{r}^{\prime \prime}\right)\left[\nabla_{l}^{\prime \prime} D_{j k}^{*}\left(\mathbf{r}^{\prime}, \mathbf{r}^{\prime \prime}\right)-\nabla_{k}^{\prime \prime} D_{j l}^{*}\left(\mathbf{r}^{\prime}, \mathbf{r}^{\prime \prime}\right)\right] \\
=-\frac{i}{\omega} \nabla_{i} \nabla_{k}^{\prime \prime}\left[D\left(\mathbf{r}, \mathbf{r}^{\prime \prime}\right)\left\{\nabla_{l}^{\prime \prime} D_{j k}^{*}\left(\mathbf{r}^{\prime}, \mathbf{r}^{\prime \prime}\right)-\nabla_{k}^{\prime \prime} D_{j l}^{*}\left(\mathbf{r}^{\prime}, \mathbf{r}^{\prime \prime}\right)\right\}\right] \\
\quad-\frac{1}{c^{2}} \nabla_{i} \nabla_{j}^{\prime} D\left(\mathbf{r}, \mathbf{r}^{\prime}\right) \nabla_{l}^{\prime \prime} D^{*}\left(\mathbf{r}^{\prime}, \mathbf{r}^{\prime \prime}\right)
\end{aligned}
$$

formula (13) gives

$$
\left\langle E_{i}(\mathbf{r}) E_{j}\left(\mathbf{r}^{\prime}\right)\right\rangle_{\omega}=\nabla_{i} \nabla_{j}^{\prime}\left\langle\phi(\mathbf{r}) \phi^{*}\left(\mathbf{r}^{\prime}\right)\right\rangle_{\omega},
$$

$$
\begin{aligned}
\left\langle\phi(\mathbf{r}) \phi^{*}\left(\mathbf{r}^{\prime}\right)\right\rangle_{\omega} & \\
= & \frac{A\left(T_{1}\right)-A\left(T_{2}\right)}{2 i} \int d \mathbf{S}_{1}^{\prime \prime}\left\{D^{*}\left(\mathbf{r}^{\prime}, \mathbf{r}^{\prime \prime}\right) \nabla^{\prime \prime} D\left(\mathbf{r}, \mathbf{r}^{\prime \prime}\right)\right. \\
& \left.-D\left(\mathbf{r}, \mathbf{r}^{\prime \prime}\right) \nabla^{\prime \prime} D^{*}\left(\mathbf{r}^{\prime}, \mathbf{r}^{\prime \prime}\right)\right\}-4 \pi A\left(T_{2}\right) \operatorname{Im} D\left(\mathbf{r}, \mathbf{r}^{\prime}\right) .
\end{aligned}
$$

\section{HEAT TRANSFER BETWEEN TWO FLAT SURFACES}

In this section we apply the general formalism to the problem of the heat transfer between two flat surfaces. This problem was considered some years ago by Polder and Van Hove, ${ }^{1}$ Levin, Polevoy, and Rytov, ${ }^{2}$ and more recently by Pendry, ${ }^{3}$ who used a completely different approach. We suppose that the half space $z<0$ is filled by a medium (temperature $\left.T_{1}\right)$ with reflection factors $R_{1 p}(\mathbf{q}, \omega)$ and $R_{1 s}(\mathbf{q}, \omega)$ for $s$ - and $p$-polarized electromagnetic fields, respectively, and the half space $z>d$ is filled by a medium (temperature $T_{2}$ ) with reflection factors $R_{2 p}(\mathbf{q}, \omega)$ and $R_{2 s}(\mathbf{q}, \omega)$, and the region between the solids, $0<z<d$, is assumed to be vacuum. Let $\mathbf{q}$ be the component of wave-vector $\mathbf{k}=(\mathbf{q}, p)$ parallel to the surfaces and

$$
p=\sqrt{\left(\frac{\omega}{c}\right)^{2}-q^{2}}
$$

We note that in our approach the calculation of the reflection factors for $s$ and $p$ waves is considered as a separate problem, which, if necessary, can be solved by taking into account nonlocal effects. Using the general formulas from Sec. II and omitting the details of calculations for the heat transfer between two semi-infinite bodies, separated by a vacuum gap with the width $d$, we obtain

$$
\begin{aligned}
S_{z}= & \frac{\hbar}{8 \pi^{3}} \int_{0}^{\infty} d \omega \omega \int_{q<\omega / c} d^{2} q \\
& \times\left[\frac{\left\{1-\left|R_{1 p}(\omega)\right|^{2}\right\}\left\{1-\left|R_{2 p}(\omega)\right|^{2}\right\}\left\{n_{1}(\omega)-n_{2}(\omega)\right\}}{\left|1-e^{2 i p d} R_{1 p}(\omega) R_{2 p}(\omega)\right|^{2}}\right] \\
& +\frac{\hbar}{2 \pi^{3}} \int_{0}^{\infty} d \omega \omega \int_{q>\omega / c} d^{2} q e^{-2|p| d} \\
& \times \frac{\operatorname{Im} R_{1 p}(\omega) \operatorname{Im} R_{2 p}(\omega)}{\left|1-e^{-2|p| d} R_{1 p}(\omega) R_{2 p}(\omega)\right|^{2}} \\
& \times\left\{n_{1}(\omega)-n_{2}(\omega)\right\}+[p \rightarrow s]
\end{aligned}
$$

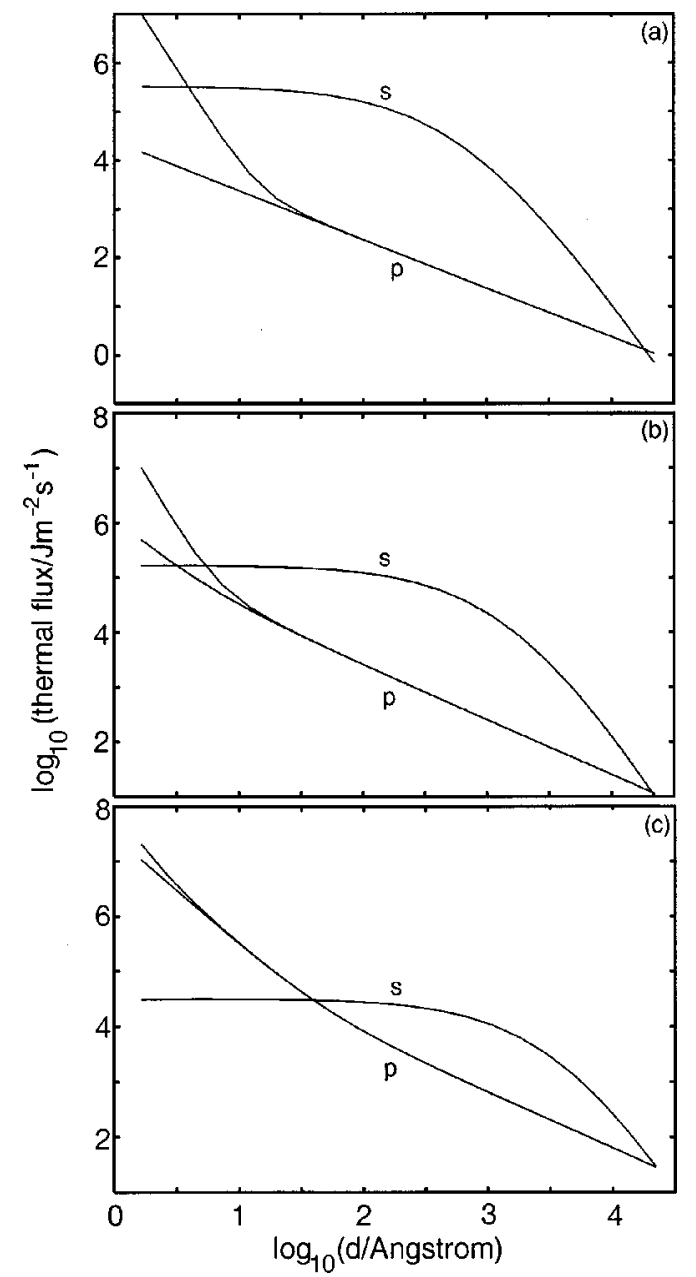

FIG. 1. (a) The heat transfer flux between two semi-infinite silver bodies as a function of the separation $d$, one at temperature $T_{1}=273 \mathrm{~K}$ and another at $T_{2}=0 \mathrm{~K}$. (b) The same as (a) except that we have reduced the Drude electron relaxation time $\tau$ for solid 1 from a value corresponding to a mean-free-path $v_{F} \tau=l=560$ to 20 $\AA$. (c) The same as (a) except that we have reduced $l$ to $3.4 \AA$.

where

$$
n_{1}(\omega)=\left(e^{\hbar \omega / k_{B} T_{1}}-1\right)^{-1}
$$

is the Bose-Einstein factor of solid 1 and similarly for $n_{2}$.

The detailed distance dependence of $S_{z}$ has been studied by Polder and Van Hove within the local optics approximation, and will not be repeated here. For the local optic case, the reflection factors are determined by the well-known Fresnel formulas

$$
R_{i p}=\frac{\varepsilon_{i} p-s_{i}}{\varepsilon_{i} p+s_{i}}, \quad R_{i s}=\frac{p-s_{i}}{p+s_{i}},
$$

where $\varepsilon_{i}$ is the complex dielectric constant for body $i$,

$$
s_{i}=\sqrt{\frac{\omega^{2}}{c^{2}} \varepsilon_{i}-q^{2}} .
$$

Figure 1(a) shows the heat transfer between two semi-infinite 


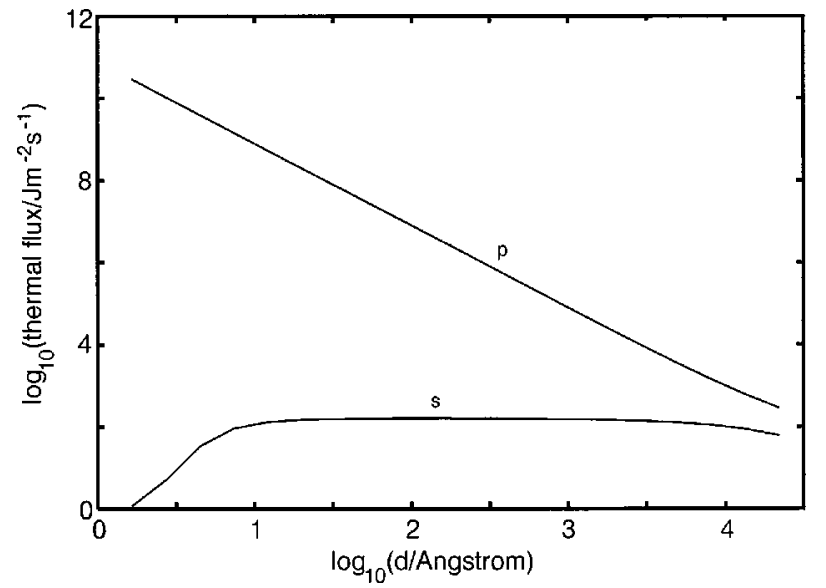

FIG. 2. The thermal flux as a function of the separation $d$ between the surfaces. One body is at zero temperature and the other at $T=273 \mathrm{~K}$. With $(\hbar / \tau) / k_{B} T=120$ and $\hbar \omega_{p} / k_{B} T=15.5$.

silver bodies separated by the distance $d$ and at the temperatures $T_{1}=273 \mathrm{~K}$ and $T_{2}=0 \mathrm{~K}$, respectively. The $s$ - and $p$-wave contributions are shown separately, and the $p$-wave contribution has been calculated using nonlocal optics (the lower curve denoted by $\mathrm{p}$ shows the result using local optics). It is remarkable how important the $s$ contribution is even for short distances. The nonlocal optics contribution to $\left(S_{z}\right)_{p}$, which is important only for $d<l$ (where $l$ is the electron mean-free path in the bulk), is easy to calculate for free-electronlike metals. The nonlocal surface contribution to $\operatorname{Im} R_{p}$ is given by ${ }^{10}$

$$
\left(\operatorname{Im} R_{p}\right)_{\text {surf }}=2 \xi \frac{\omega}{\omega_{p}} \frac{q}{k_{F}}
$$

where $\xi(q)$ depends on the electron-density parameter $r_{s}$ but typically $\xi(0) \sim 1$. Using this expression for $\operatorname{Im} R_{p}$ in Eq. (23) gives the (surface) contribution:

$$
S_{\mathrm{surf}} \approx \frac{\xi^{2} k_{B}^{4}}{\omega_{p}^{2} k_{F}^{2} d^{4} \hbar^{3}}\left(T_{1}^{4}-T_{2}^{4}\right) .
$$

Note from Fig. 1(a) that the local optics contribution to $S_{p z}$ depends nearly linearly on $1 / d$ in the distance interval studied, and that this contribution is much smaller than the $s$-wave contribution. Both these observations differ from Ref. 3, where it is stated that the $s$ contribution can be neglected for small distances and that the $p$-wave contribution (within local optics) is proportional to $1 / d^{2}$ for small distances. However, for very high-resistivity materials, the $p$-wave contribution becomes much more important, and a crossover to a $1 / d^{2}$ dependence of $S_{p z}$ is observed at short separations $d$. This is illustrated in Figs. 1(b) and 1(c) which have been calculated with the same parameters as in Fig. 1(a), except that the electron mean-free path has been reduced from $l=560 \AA$ (the electron mean-free path for silver at room temperature) to $20 \AA$ (roughly the electron meanfree path in lead at room temperature) [Fig. 1(b)] and $3.4 \AA$ (of order the lattice constant, representing the minimal possible mean-free path) [Fig. 1(c)]. Note that when $l$ decreases,

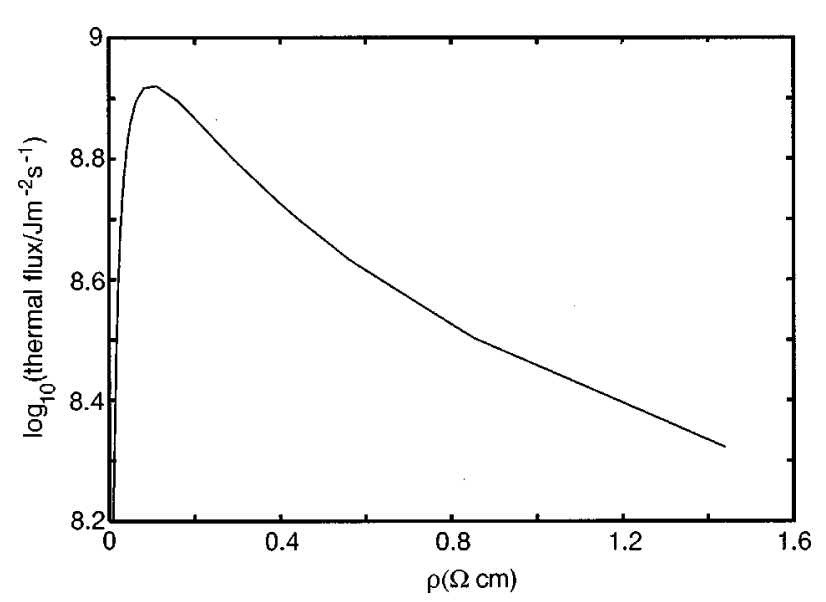

FIG. 3. The thermal flux as a function of the resistivity of the solids. The solid surfaces are separated by $d=10 \AA$ and $\hbar \omega_{p}$ $\gg k_{B} T$. The heat flux for other separations can be obtained using scaling $\sim 1 / d^{2}$ that holds for high-resistivity materials.

the $p$ contribution to the heat transfer increases while the $s$ contribution decreases. Since the mean-free path cannot be much smaller than the lattice constant, the result in Fig. 1(c) represent the largest possible $p$-wave contribution for normal metals. However, the $p$-wave contribution may be even larger for other materials, e.g., semimetals, with lower carrier concentration than in normal metals. This fact has already been pointed out by Pendry: the $p$-wave contribution for short distances is expected to be maximal when the function

$$
\operatorname{Im} R_{p} \approx \operatorname{Im} \frac{\varepsilon-1}{\varepsilon+1}=\operatorname{Im}\left[1-2 \frac{\omega}{\omega_{p}}\left(\frac{\omega}{\omega_{p}}+\frac{i}{\omega_{p} \tau}\right)\right]^{-1}
$$

is maximal with respect to variations in $1 / \tau$. This gives

$$
\omega_{p} \tau=\frac{2 k_{B} T}{\hbar \omega_{p}}
$$

where we have used that typical frequencies $\omega \sim k_{B} T / \hbar$. Since the dc resistivity $\rho=4 \pi /\left(\omega_{p}^{2} \tau\right)$ we get (at room temperature) $\rho \approx 2 \pi \hbar / k_{B} T \approx 0.14 \Omega \mathrm{cm}$. To illustrate this case, Fig. 2 shows the thermal flux as a function of the separation $d$ between the surfaces when $(\hbar / \tau) / k_{B} T=120$ and $\hbar \omega_{p} / k_{B} T=15.5$. One body is at zero temperature and the other at $T=273 \mathrm{~K}$.

Figure 3 shows the thermal flux as a function of the resistivity of the solids. Again we assume that one body is at zero temperature and the other at $T=273 \mathrm{~K}$. The solid surfaces are separated by $d=10 \AA$ and $\hbar \omega_{p} \gg k_{B} T$. The heat flux for other separations can be obtained using scaling $\sim 1 / d^{2}$, which holds for high-resistivity materials. Finally, we note that thin high-resistivity coatings can drastically increase the heat transfer between two solids. This is illustrated in Fig. 4, which shows the heat flux when thin films $(\sim 10 \AA)$ of high-resistivity material $\rho=0.14 \Omega \mathrm{cm}$, are deposited on silver. One body is at zero temperature and the other at $T$ $=273 \mathrm{~K}$. (a) and (b) shows the $p$ and $s$ contributions, respectively. Also shown are the heat flux when the two bodies are 


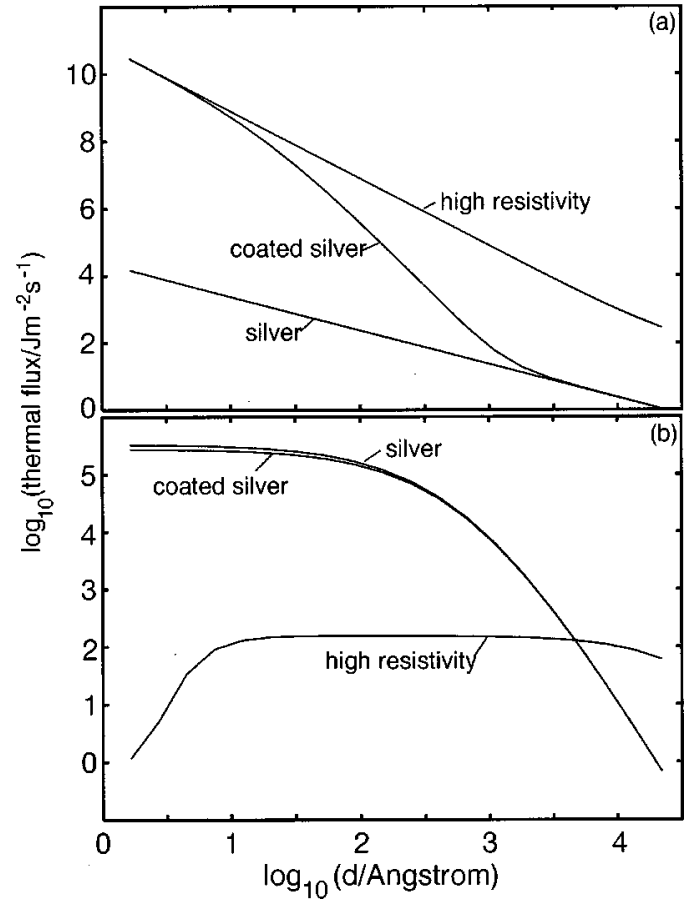

FIG. 4. The heat flux between two semi-infinite silver bodies coated with $10 \AA$ high-resistivity $(\rho=0.14 \Omega \mathrm{cm})$ material. Also shown is the heat flux between two silver bodies, and two highresistivity bodies. One body is at zero temperature and the other at $T=273 \mathrm{~K}$. (a) and (b) show the $p$ and $s$ contributions, respectively.

made from silver, and from the high-resistivity material. It is interesting to note that while the $p$ contribution to the heat flux for the coated surfaces is strongly influenced by the coating, the $s$ contribution is nearly unaffected.

\section{LOCAL HEATING OF A SURFACE BY AN STM TIP}

It was pointed by Pendry ${ }^{3}$ the local heating of a surface by an STM tip can be used for local modification of a surface if the heat transfer is sufficiently great. To investigate the power of a hot tip to heat a surface, Pendry modeled the tip as a hot sphere of the same radius $R$ as the tip. This is a common approximation when calculating tunneling current and the same arguments justify its use for calculating heat tunneling. Pendry considered the case $R \ll d \ll d_{W} \sim c \hbar / k_{B} T$ and the electrostatic limit. However, for an STM tip, an opposite limit $d \ll R$ is usually realized, and at large distances retardation effects can be important. In fact, it will be shown below that the heat transfer between a sphere and surface in the asymptotic limit (large separation) can be obtained directly from formula (23).

Consider distances $d \ll d_{W} \sim c \hbar / k_{B} T$ (at $T=300 \mathrm{~K}$ we have $d_{W} \sim 10^{5} \AA$ ). In this case we can neglect the first integral in Eq. (23), and in the second integral we can put $p$ $\approx i q$, and extend the integral to the whole $q$ plane. Using these approximations, the second integral in Eq. (23) can be written as

$$
\begin{aligned}
S_{z}= & \frac{\hbar}{\pi^{2}} \int_{0}^{\infty} d \omega \omega\left[n_{1}(\omega)-n_{2}(\omega)\right] \int_{0}^{\infty} d q q e^{-2 q d} \\
& \times\left\{\frac{\operatorname{Im} R_{1 p}(\omega) \operatorname{Im} R_{2 p}(\omega)}{\left|1-e^{-2 q d} R_{1 p}(\omega) R_{2 p}(\omega)\right|^{2}}+[p \rightarrow s]\right\} .
\end{aligned}
$$

Now, assume that the medium $\mathbf{2}$ is sufficiently rarefied and consists of particles with the radius $R \ll d$, and the polarizability $\alpha(\omega)$. Then, $\varepsilon_{2}-1 \rightarrow 4 \pi \alpha_{2} n \ll 1$, where $n$ is the number of particles per unit volume. Thus, when $n \rightarrow 0$ it is enough to include only the first-nonvanishing terms in the expansion of the integrand of Eq. (27) in powers of $\varepsilon_{2}-1$. The heat transfer between one particle and a surface can be obtained as the ratio between the change of heat transfer after displacement of body 2 by small distance $d z$, and the number of particles in a slab with thickness $d z$ :

$$
\begin{aligned}
S_{z}= & 2 \frac{\hbar}{\pi} \int_{0}^{\infty} d \omega \omega\left[n_{1}(\omega)-n_{2}(\omega)\right] \int_{0}^{\infty} d q q^{2} e^{-2 q d} \\
& \times\left\{2 \operatorname{Im} R_{1 p}(\omega) \operatorname{Im} \alpha_{2}(\omega)\right. \\
& \left.+\left(\frac{\omega}{c q}\right)^{2} \operatorname{Im} R_{1 s}(\omega) \operatorname{Im} \alpha_{2}(\omega)\right\} .
\end{aligned}
$$

In the comparison with the Pendry's calculations formula, Eq. (28) includes the $s$-wave contribution that is given by the second term.

To simplify this expression we assume that $|\varepsilon(\omega)| \gg 1$ holds for all relevant frequencies. In the limit $d$ $<|\varepsilon|^{-1 / 2} d_{W}$, where $\varepsilon$ is taken at the characteristic frequency $\sim k_{B} T / \hbar$, the reflection factor of the $p$ wave becomes

$$
R_{1 p} \approx \frac{\varepsilon_{1}-1}{\varepsilon_{1}+1}, \operatorname{Im} R_{1 p} \approx \frac{2 \operatorname{Im} \varepsilon_{1}}{\left|\varepsilon_{1}\right|^{2}} .
$$

The polarizability of the sphere is determined by

$$
\alpha_{2}=\frac{\varepsilon_{2}-1}{\varepsilon_{2}+2} R^{3}, \quad \operatorname{Im} \alpha_{2} \approx \frac{3 \operatorname{Im} \varepsilon_{2}}{\left|\varepsilon_{2}\right|^{2}} .
$$

We describe the sphere the same dielectric function as the substrate:

$$
\varepsilon(\omega)=1-\frac{\omega_{p}^{2}}{\omega(\omega+i / \tau)},
$$

where $\tau$ is the Drude relaxation time and $\omega_{p}$ the plasma frequency. In this case the $p$-wave contribution becomes

$$
S_{p z} \approx \frac{2 \pi^{3} R^{3} k_{B}^{4}}{5 d^{3} \hbar^{3}} \frac{1}{\left(\omega_{p}^{2} \tau\right)^{2}}\left(T_{1}^{4}-T_{2}^{4}\right) .
$$

This result is in agreement with calculations of Pendry. ${ }^{3}$ To evaluate the $s$-wave contribution in the limit $d<|\varepsilon|^{-1 / 2} d_{W}$, we use the integral 


$$
\begin{aligned}
& \int_{0}^{\infty} d q e^{-2 q d} \operatorname{Im} \frac{i q-s}{i q+s} \\
& \quad \approx \int_{0}^{\infty} d q \operatorname{Im} \frac{i q-s}{i q+s} \\
& \quad=\operatorname{Im}\left\{\left|s_{0}\right| e^{i \phi} \int_{0}^{\infty} d t \frac{t-\sqrt{t^{2}-1}}{t+\sqrt{t^{2}-1}}\right\} \\
& \quad=\frac{1}{2} \operatorname{Im}\left\{\left|s_{0}\right| e^{i \phi} \int_{-i \pi / 2}^{\infty} d z\left(e^{-z}-e^{-3 z}\right)\right\} \\
& \quad=\frac{2}{3}\left|s_{0}\right| \cos (\phi),
\end{aligned}
$$

where $s_{0}=s(q=0)=(\omega / c) \sqrt{\varepsilon},\left|s_{0}\right|^{1 / 2}, \phi=\arg s_{0}$. Thus,

$$
S_{s z} \approx 2 \cdot 10^{2} k_{B}^{11 / 2} h^{-9 / 2} R^{3} c^{-3} T^{11 / 2}\left(\omega_{p}^{2} \tau\right)^{-1 / 2} .
$$

From the comparison (32) and (33) the $s$-wave contribution exceeds the $p$-wave contribution for $d>\left(d_{W} c / \omega_{p}^{2} \tau\right)^{1 / 2}$; for typical metals at room temperature, $\hbar \omega_{p} / k_{B} T \sim 10^{3}$ and $\omega_{p} \tau \sim 10^{2}$ so that $d>10^{2} \AA$. For $d_{W}|\varepsilon|^{-1 / 2}<d<d_{W}$ we obtain

$$
S_{p z} \approx S_{s z} \approx 10 k_{B}^{9 / 2} \hbar^{-7 / 2} d^{-2} R^{3} c^{-1} T^{9 / 2}\left(\omega_{p}^{2} \tau\right)^{-3 / 2} .
$$

Assume now that the spherical particle is so close to the surface that we can neglect retardation effects. In this case the problem is reduced to the finding of electrostatic potential created by a point charge located in vacuum. Using the image theorem, ${ }^{11}$ the electrostatic potential can be written in the form

$$
\begin{aligned}
D\left(\mathbf{r}, \mathbf{r}^{\prime}\right)= & \frac{1}{\left|\mathbf{r}-\mathbf{r}^{\prime}\right|}-\frac{\varepsilon_{1}-1}{\varepsilon_{1}+1} \frac{1}{\left|\mathbf{r}-\widetilde{\mathbf{r}}^{\prime}\right|}+\sum_{m=0}^{\infty} \sum_{n=m}^{\infty} C_{n}^{m}\left(\mathbf{r}^{\prime}\right) \\
& \times\left(\frac{P_{n}^{m}(\cos \theta)}{r^{n+1}}-(-1)^{n+m} \frac{\varepsilon_{1}-1}{\varepsilon_{1}+1} \frac{P_{n}^{m}\left(\cos \theta_{i}\right)}{r_{i}^{n+1}}\right) \\
& \times \cos m\left(\phi-\phi^{\prime}\right),
\end{aligned}
$$

where we have chosen the origin of the coordinate system at the center $O$ of the spherical particle, and taken the polar axis along the line connecting $O$ with the center of "image" sphere $O^{\prime}$, and assumed that the points $\mathbf{r}$ and $\mathbf{r}^{\prime}$ have the polar coordinates $(r, \theta, \phi)$ and $\left(r^{\prime}, \theta^{\prime}, \phi^{\prime}\right)$ with respect $O ; \widetilde{\mathbf{r}}^{\prime}$ is the "image" of $\mathbf{r}^{\prime} ; \mathbf{r}_{i}=\mathbf{r}-\mathbf{h}=\left(r_{i}, \theta_{i}, \phi\right)$ where $\mathbf{h}$ is the vector connecting the centers $O$ and $O^{\prime} . h=2(R+d)$ and $P_{n}^{m}(\cos \theta)$ is the associated Legendre function (see Fig. 5). At any interior point of the sphere, the resultant potential is

$$
\begin{gathered}
D\left(\mathbf{r}, \mathbf{r}^{\prime}\right)=\sum_{m=0}^{\infty} \sum_{n=m}^{\infty} A_{n}^{m}\left(\mathbf{r}^{\prime}\right) r^{n} P_{n}^{m}(\cos \theta) \cos m\left(\phi-\phi^{\prime}\right), \\
(r<R) .
\end{gathered}
$$

We expand the potential (35) in spherical harmonics around the center $O$ using the formulas ${ }^{12}$

$$
\begin{aligned}
\frac{1}{\left|\mathbf{r}-\mathbf{r}^{\prime}\right|}= & \sum_{m=0}^{\infty} \sum_{n=m}^{\infty} \frac{r^{n}}{r^{\prime n+1}} \frac{(n-m) !}{(n+m) !} P_{n}^{m}(\cos \theta) \\
& \times P_{n}^{m}\left(\cos \theta^{\prime}\right) \boldsymbol{\epsilon}_{m} \cos m\left(\phi-\phi^{\prime}\right), \quad\left(r<r^{\prime}\right), \\
\frac{1}{\left|\mathbf{r}-\mathbf{r}^{\prime}\right|}= & \sum_{m=0}^{\infty} \sum_{n=m}^{\infty} \sum_{l=m}^{\infty}(-1)^{l+n} \frac{r^{n} r^{\prime l}}{h^{l+n+1}} \frac{(l+n) !}{(l+m) !(n+m) !} \\
& \times P_{n}^{m}(\cos \theta) P_{l}^{m}\left(\cos \theta^{\prime}\right) \boldsymbol{\epsilon}_{m} \cos m\left(\phi-\phi^{\prime}\right), \\
(-1)^{n+m} & \frac{P_{n}^{m}\left(\cos \theta_{i}\right)}{r_{i}^{n+1}} \quad\left(r, r^{\prime}<h / 2\right), \\
=\sum_{l=m}^{\infty} & (-1)^{l+n} \frac{r^{l}}{h^{n+l+1}}\left(\begin{array}{l}
l+n \\
l+m
\end{array}\right) P_{l}^{m}(\cos \theta), \quad(r<h),
\end{aligned}
$$

where $\epsilon_{0}=1, \epsilon_{m}=2$ for $m \neq 0$. Using Eqs. (37)-(39) we can rewrite Eq. (35) in the form

$$
\begin{aligned}
D\left(\mathbf{r}, \mathbf{r}^{\prime}\right)= & \sum_{m=0}^{\infty} \sum_{n=m}^{\infty} \sum_{l=m}^{\infty}\left\{r ^ { n } \left(\frac{1}{r^{\prime n+1}} \frac{(n-m) !}{(n+m) !} \delta_{n l}\right.\right. \\
& \left.+(-1)^{l+n+1} \frac{\varepsilon_{1}-1}{\varepsilon_{1}+1} \frac{r^{\prime l}}{h^{l+n+1}} \frac{(l+n) !}{(l+m) !(n+m) !}\right) \\
& \times P_{n}^{m}(\cos \theta) P_{l}^{m}\left(\cos \theta^{\prime}\right)+C_{n}^{m}\left(\mathbf{r}^{\prime}\right) \\
& \times\left[\frac{\delta_{n l}}{r^{n+1}}+(-1)^{l+n+1} \frac{\varepsilon_{1}-1}{\varepsilon_{1}+1} \frac{r^{l}}{h^{l+n+1}}\left(\begin{array}{c}
l+n \\
l+m
\end{array}\right)\right] \\
& \left.\times P_{l}^{m}(\cos \theta)\right\} \boldsymbol{\epsilon}_{m} \cos m\left(\phi-\phi^{\prime}\right) .
\end{aligned}
$$

Across the surface of the sphere, the Green function $D\left(\mathbf{r}, \mathbf{r}^{\prime}\right)$ (as a function of $\mathbf{r}$ ), and its normal derivatives must be continuous. These boundary conditions lead to the equation

$$
\begin{aligned}
C_{n}^{m}\left(\mathbf{r}^{\prime}\right)= & A_{n} \sum_{l=m}^{\infty}\left\{\left(\frac{1}{r^{\prime n+1}} \frac{(n-m) !}{(n+m) !} \delta_{n l}+(-1)^{l+n+1}\right.\right. \\
& \left.\times \frac{\varepsilon_{1}-1}{\varepsilon_{1}+1} \frac{r^{\prime l}}{h^{l+n+1}} \frac{(l+n) !}{(l+m) !(n+m) !}\right) P_{l}^{m}\left(\cos \theta^{\prime}\right) \\
& \left.+\frac{\varepsilon_{1}-1}{\varepsilon_{1}+1}\left(-\frac{1}{h}\right)^{l+n+1}\left(\begin{array}{c}
n+l \\
n+m
\end{array}\right) C_{l}^{m}\left(\mathbf{r}^{\prime}\right)\right\} \\
A_{n} & =\frac{\left(1-\varepsilon_{2}\right) n}{n \varepsilon_{2}+n+1} R^{2 n+1}
\end{aligned}
$$

Outside the sphere the function $C_{n}^{m}\left(\mathbf{r}^{\prime}\right)$ satisfies the Laplace equation and can be expanded as

$$
C_{n}^{m}\left(\mathbf{r}^{\prime}\right)=\sum_{l=m}^{\infty}\left(\frac{a_{n l}^{m}}{r^{\prime l+1}}+b_{n l}^{m} r^{\prime l}\right) P_{l}^{m}\left(\cos \theta^{\prime}\right) .
$$




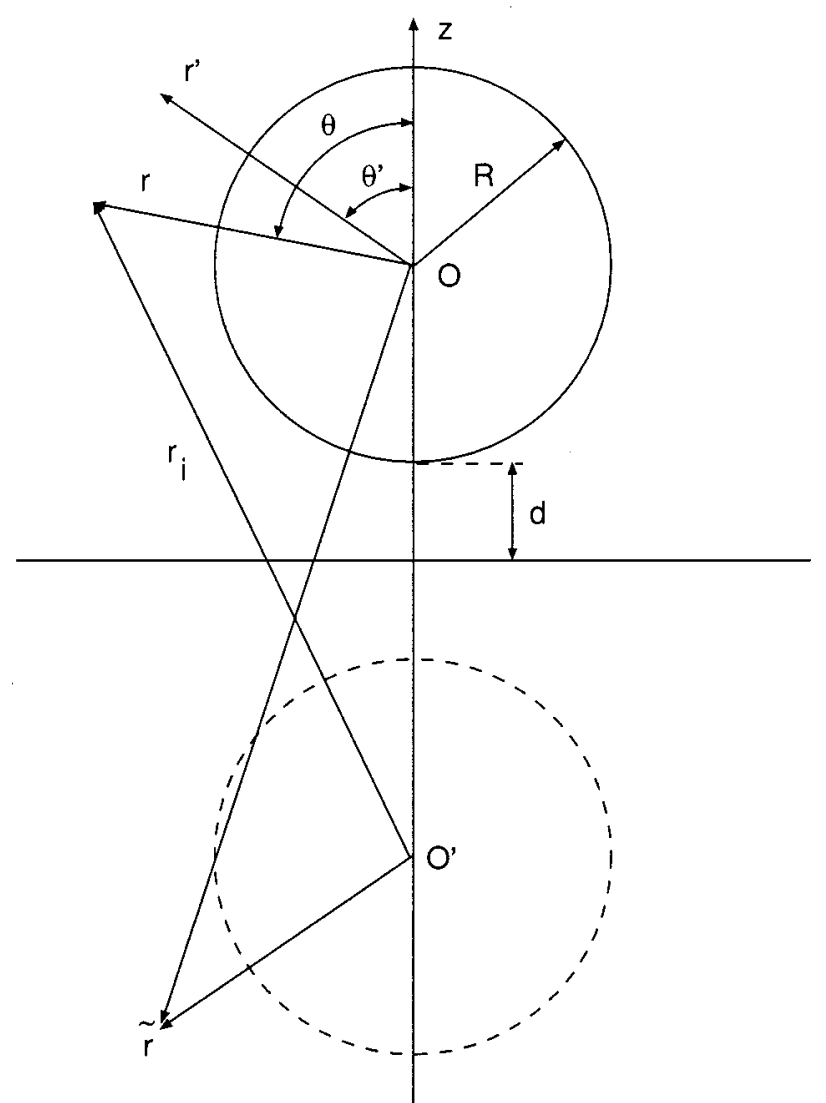

FIG. 5. Spherical particle (origin $O$ ) above a flat surface and its "image", (origin $O^{\prime}$ ).

Using Eq. (43) in Eq. (41) and taking into account that Eq. (41) must be satisfied at arbitrary $r^{\prime}$ we obtain equations for coefficients $a_{n l}^{m}$ and $b_{n l}^{m}$

$$
\begin{aligned}
a_{n l}^{m}= & A_{n} \sum_{l^{\prime}=m}^{\infty}\left\{\frac{(n-m) !}{(n+m) !} \delta_{n l^{\prime}} \delta_{l l^{\prime}}+\frac{\varepsilon_{1}-1}{\varepsilon_{1}+1}\right. \\
& \left.\times\left(-\frac{1}{h}\right)^{l^{\prime}+n+1}\left(\begin{array}{l}
n+l^{\prime} \\
n+m
\end{array}\right) a_{l^{\prime} l}^{m}\right\}, \\
b_{n l}^{m}= & A_{n} \frac{\varepsilon_{1}-1}{\varepsilon_{1}+1} \sum_{l^{\prime}=m}^{\infty}\left(-\frac{1}{h}\right) \\
& \times\left[\frac{\left(l^{\prime}+n\right) !}{\left(l^{\prime}+m\right) !(n+m) !} \delta_{l l^{\prime}}+\left(\begin{array}{l}
n+l^{\prime} \\
n+m
\end{array}\right) b_{l^{\prime} l}^{m}\right]
\end{aligned}
$$

If we make the replacement

$$
a_{n l}^{m}=A_{n} \frac{(n-m) !}{(n+m) !} \delta_{n l}+\widetilde{a}_{n l}^{m}
$$

then from Eqs. (44) and (45) we obtain

$$
b_{n l}^{m}=\frac{\widetilde{a}_{n l}^{m}}{A_{l}}
$$

Let us introduce the dimensionless coefficients $x_{n l}$

$$
\begin{aligned}
\tilde{a}_{n l}^{m} & =\left(-\frac{1}{h}\right)^{l+n+1} \frac{R_{1 p}}{(n+m) !(l+m) !} A_{n} A_{l} x_{n l} \\
& =(-\xi R)^{n+l+1} \frac{R_{1 p}}{(n+m) !(l+m) !} \lambda_{n} \lambda_{l} x_{n l},
\end{aligned}
$$

where

$$
R_{i p}=\frac{\varepsilon_{i}-1}{\varepsilon_{i}+1}
$$

$\xi=(R / h), \lambda_{n}=-A_{n} / R^{2 n+1}$. The coefficients $x_{n l}$ obey the equation

$$
x_{n l}=(n+m) !+R_{1 p} \sum_{l^{\prime}=m}^{\infty} \xi^{2 l^{\prime}+1} \lambda_{l^{\prime}} \frac{\left(n+l^{\prime}\right) !}{\left(l^{\prime}-m\right) !\left(l^{\prime}+m\right) !} x_{l^{\prime} l} \text {. }
$$

The solution of Eq. (49) can be found by iterations and has the form

$$
\begin{aligned}
x_{n l}= & (n+m) !+R_{1 p} \sum_{l^{\prime}=m}^{\infty} \xi^{2 l^{\prime}+1} \lambda_{l^{\prime}} \frac{\left(n+l^{\prime}\right) !\left(l^{\prime}+l\right) !}{\left(l^{\prime}-m\right) !\left(l^{\prime}+m\right) !} \\
& +R_{1 p}^{2} \sum_{l^{\prime}=m}^{\infty} \sum_{l^{\prime \prime}=m}^{\infty} \xi^{2 l^{\prime}+1} \xi^{2 l^{\prime \prime}+1} \lambda_{l^{\prime}} \lambda_{l^{\prime \prime}} \\
& \times \frac{\left(n+l^{\prime}\right) !\left(l^{\prime}+l\right) !}{\left(l^{\prime}-m\right) !\left(l^{\prime}+m\right) !} \frac{\left(l^{\prime}+l^{\prime \prime}\right) !\left(l^{\prime}+l\right) !}{\left(l^{\prime \prime}-m\right) !\left(l^{\prime \prime}+m\right) !}+\cdots
\end{aligned}
$$

Using Eqs. (44) and (45), formula (40) can be significantly simplified

$$
\begin{aligned}
D\left(\mathbf{r}, \mathbf{r}^{\prime}\right)= & \left.\sum_{m=0}^{\infty} \sum_{l=m}^{\infty} C_{l}^{m}\left(\mathbf{r}^{\prime}\right)\left(\frac{1}{r^{l+1}}+\frac{r^{l}}{A_{l}}\right) P_{l}^{m}(\cos \theta)\right\} \\
& \times \epsilon_{m} \cos m\left(\phi-\phi^{\prime}\right) .
\end{aligned}
$$

Using Eq. (51) in Eq. (21) we obtain

$$
\begin{aligned}
\langle\phi(\mathbf{r}) & \left.\phi^{*}\left(\mathbf{r}^{\prime}\right)\right\rangle_{\omega} \\
= & \frac{\hbar}{\pi}\left[n_{1}(\omega)-n_{2}(\omega)\right] \sum_{m=0}^{\infty} \sum_{n=m}^{\infty} \\
& \times\left\{\frac{(n+m) !}{(n-m) !} \frac{c_{n}^{m}(\mathbf{r}) C_{n}^{* m}\left(\mathbf{r}^{\prime}\right)}{R^{2 n+1}} \frac{\operatorname{Im} \lambda_{n}}{\left|\lambda_{n}\right|^{2}} \cos m\left(\phi-\phi^{\prime}\right)\right\} \\
& -4 \pi A\left(T_{1}\right) \operatorname{Im} D\left(\mathbf{r}, \mathbf{r}^{\prime}\right) .
\end{aligned}
$$

Using Eq. (52) in Eq. (17) for the heat transfer between a sphere and a flat surface we obtain 


$$
\begin{aligned}
S= & \frac{\hbar}{\pi^{2}} \int_{0}^{\infty} d \omega \omega\left[n_{1}(\omega)-n_{2}(\omega)\right] \\
& \times \sum_{m=0}^{\infty} \sum_{n=m}^{\infty} \sum_{l=m}^{\infty} \frac{\xi^{n+l+1}}{(n-m) !(l-m) !} \\
& \times\left((n-m) \operatorname{Im}\left(\widetilde{x}_{n n}^{m} R_{1 p}^{*}\right) \delta_{n l}\right. \\
& \left.-\frac{\xi^{n+l+1}\left|R_{1 p}\right|^{2}}{(n+l) !(l+m) !}\left|\tilde{x}_{l n}^{m}\right|^{2} \operatorname{Im} \lambda_{l}\right) \operatorname{Im} \lambda_{n} .
\end{aligned}
$$

The above formalism gives, in principle, an exact solution of the problem in the nonretarded limit. However, for $d \ll R$, extensive numerical calculations are necessary, because in this case the series converges slowly. The numerical results will be presented elsewhere. In the present paper we only present an approximate solution of the problem.

Using the image theorem for $\varepsilon \gg 1$ and for the points $\mathbf{r}$ and $\mathbf{r}^{\prime}$ close to the surface of the sphere, in the first approximation in the expansion of the electrostatic potential in the sum of the potentials created by the image charges we can write the potential in the form

$$
\begin{aligned}
D\left(\mathbf{r}, \mathbf{r}^{\prime}\right)= & \frac{1}{\left|\mathbf{r}-\mathbf{r}^{\prime}\right|}-R_{1 p} \frac{1}{\left|\mathbf{r}-\widetilde{\mathbf{r}}^{\prime}\right|}-R_{2 p} \frac{1}{\left|\mathbf{r}-\mathbf{r}_{i}^{\prime}\right|} \\
& +R_{1 p} R_{2 p} \frac{1}{\left|\mathbf{r}-\widetilde{\mathbf{r}}_{i}^{\prime}\right|},
\end{aligned}
$$

where

$$
\mathbf{r}_{i}^{\prime}=\frac{2 R-r^{\prime}}{r^{\prime}}\left(x^{\prime}, y^{\prime}, z^{\prime}\right)
$$

$\mathbf{r}^{\prime}=\left(x^{\prime}, y^{\prime},-h-z^{\prime}\right)$ and $\widetilde{\mathbf{r}}_{i}^{\prime}=\left(x_{i}^{\prime}, y_{i}^{\prime},-h-z_{i}^{\prime}\right)$. The value of the surface integral (21) does not change if we assume that the potential has the form (54) in all space outside a sphere. Thus, using Green's theorem we can convert the surface integral to a volume integral over all space outside a sphere. This volume integral can be easily calculated using the fact that outside a sphere the potential $D\left(\mathbf{r}, \mathbf{r}^{\prime}\right)$ obeys Poisson's equation with the point charges located at $\mathbf{r}=\mathbf{r}^{\prime}, \mathbf{r}=\widetilde{\mathbf{r}}^{\prime}$, and $\mathbf{r}=\widetilde{\mathbf{r}}_{i}^{\prime}$. Performing the calculation gives

$$
\begin{aligned}
\operatorname{Im}\left\langle\phi(\mathbf{r}) \phi^{*}\left(\mathbf{r}^{\prime}\right)\right\rangle_{\omega} & \\
= & \frac{\hbar}{2 \pi}\left[n_{1}(\omega)-n_{2}(\omega)\right] \operatorname{Re}\left\{R_{1 p} R_{2 p}^{*} \frac{1}{\left|\mathbf{r}_{i}^{\prime}-\widetilde{\mathbf{r}}\right|}-R_{1 p}^{*} R_{2 p}\right. \\
& \left.\times \frac{1}{\left|\widetilde{\mathbf{r}}^{\prime}-\mathbf{r}_{i}\right|}+R_{1 p} R_{2 p} \frac{1}{\left|\mathbf{r}^{\prime}-\widetilde{\mathbf{r}}_{i}\right|}-R_{1 p}^{*} R_{2 p}^{*} \frac{1}{\left|\widetilde{\mathbf{r}}_{i}^{\prime}-\widetilde{\mathbf{r}}\right|}\right\} .
\end{aligned}
$$

Using Eq. (55) in Eq. (17) for the heat transfer between a sphere and a flat surface we obtain

$$
\begin{aligned}
S= & \frac{\hbar}{2 \pi} \int_{0}^{\infty} d \omega \omega\left[n_{1}(\omega)-n_{2}(\omega)\right] \operatorname{Im} R_{1 p}(\omega) \operatorname{Im} R_{2 p}(\omega) \\
& \times \int_{0}^{\pi} d \theta \frac{\cos ^{3} \theta \sin \theta}{\left[(2 \xi)^{-1}-\cos \theta\right]^{2}} \\
\approx & \frac{\pi}{30} \frac{R}{d} \sigma_{1}^{-1} \sigma_{2}^{-1} \hbar^{-3} k_{B}^{4}\left(T_{1}^{4}-T_{2}^{4}\right)
\end{aligned}
$$

where $\sigma=\omega_{p}^{2} \tau / 4 \pi$. If we assume that in an accordance with Eq. (32) every elementary volume of the sphere gives the contribution to the heat transfer

$$
\begin{aligned}
d S_{p z}(r, \theta, \phi) \approx & \frac{3 k_{B}^{4}}{160(r+d-r \cos \theta)^{3} \hbar^{3}} \\
& \times \sigma_{1}^{-1} \sigma_{2}^{-1}\left(T_{1}^{4}-T_{2}^{4}\right) d V
\end{aligned}
$$

then after integration of Eq. (57) over the volume of the sphere, we obtain the result of the same order magnitude as Eq. (56). Because for $d<R<d_{W}|\varepsilon|^{-1 / 2}$ in the accordance with Eq. (33) the $s$ contribution of the small particle does not depend on the separation $d$ formula (33) is valid also for small separation $d$. From the comparison of Eqs. (56) and (33), we get that for the sphere close to the surface, the $s$-wave contribution dominates for

$$
d>10^{-3}\left(d_{W} c / \omega_{p}\right)^{3 / 2} R^{-2}\left(\omega_{p} \tau\right)^{-3 / 2} .
$$

For "normal" metals at room temperature and for $R$ $\sim 10^{3} \AA$ the $s$-wave contribution dominates for $d>1 \AA$.

\section{HEATING OF A PARTICLE BY AN STM TIP}

Let us now consider the heat transfer between an STM tip, which we again model by a spherical particle with radius $R_{2}$ and the polarizability $\alpha_{2}(\omega)$, and a spherical particle with radius $R_{1}$ and the polarizability $\alpha(\omega)$ located on a surface. We consider the case of large separation $d \gg R_{1}, R_{2}$, and neglect by influence of the substrate on the heat transfer. At large distances, the thermal electromagnetic field radiated by particle 1 can be considered as the radiative electromagnetic field of a fluctuating point dipole $\mathbf{p}^{f}$ with ensemble average

$$
\left\langle p_{i}^{f} p_{k}^{f *}\right\rangle=A\left(T_{1}\right) \operatorname{Im} \alpha_{1}(\omega) \delta_{i k} .
$$

The electric field of this point dipole is given by

$$
\mathbf{E}=\left[3 \mathbf{n}\left(\mathbf{n} \cdot \mathbf{p}^{f}\right)-\mathbf{p}^{f}\right]\left(\frac{1}{r^{3}}-\frac{i k}{r^{2}}\right) e^{i k}-k^{2}\left[\mathbf{n}\left(\mathbf{n} \cdot \mathbf{p}^{f}\right)-\mathbf{p}^{f}\right] \frac{e^{i k r}}{r}
$$

where $k=\omega / c$ and where $\mathbf{n}$ is a unit vector along the axis connecting the two particle. The rate at which a particle $\mathbf{1}$ does work on a particle $\mathbf{2}$ is determined by

$$
P=4 \int_{0}^{\infty} d \omega \omega \operatorname{Im} \alpha_{2}(\omega)\left\langle E_{i} E_{i}^{*}\right\rangle_{\omega}
$$




$$
\left\langle E_{i} E_{i}^{*}\right\rangle_{\omega}=A\left(T_{1}, \omega\right) \operatorname{Im} \alpha_{1}(\omega)\left(\frac{6}{d^{6}}+\frac{2 k^{2}}{d^{4}}+\frac{2 k^{4}}{d^{2}}\right) .
$$

After absorption by particle 2 this work is converted into heat. In the same manner we can calculate the rate of cooling of particle 2 using the same formula by reciprocity. Thus, the total heat transfer between the particles will determined by

$$
\begin{aligned}
S= & \frac{\hbar}{(2 \pi)^{2}} \int_{0}^{\infty} d \omega \omega\left[n_{1}(\omega)-n_{2}(\omega)\right] \operatorname{Im} \alpha_{1}(\omega) \operatorname{Im} \alpha_{2}(\omega) \\
& \times\left(\frac{6}{d_{6}}+\frac{2 k^{2}}{d^{4}}+\frac{2 k^{4}}{d^{2}}\right) .
\end{aligned}
$$

Using for the polarizabilities of particles expression (30) we obtain

$$
\begin{aligned}
S \approx & 10^{-} \hbar^{-3} k_{B}^{4} T_{1}^{4} R_{1}^{3} R_{2}^{3} \sigma_{1}^{-1} \sigma_{2}^{-1} \\
& \times\left(\frac{6}{d^{6}}+2 \pi^{2} \frac{1}{d_{1 W}^{2} d^{4}}+8 \pi^{4} \frac{1}{d_{1 W}^{4} d^{2}}\right)-\left[T_{1} \rightarrow T_{2}\right],
\end{aligned}
$$

where $d_{i W}=\hbar c / k_{B} T_{i}$.

\section{RELATION BETWEEN HEAT TRANSFER AND FRICTION}

The heat transfer studied above is closely related to the frictional stress between bodies in relative motion, separated by a vacuum gap. In the last years, this "vacuum" friction has attracted a great deal of attention in connection with the development of the scanning probe technique. ${ }^{7,10,13-16}$ In Ref. 7 we show that the frictional stress between bodies having flat parallel surfaces separated by a distance $d$ and moving with velocity $V$ relative to each other for the distances $d \ll d_{W}$ is determined by a formula that is very similar to Eq. (27):

$$
\begin{aligned}
\sigma= & \frac{\hbar V}{2 \pi^{2}} \int_{0}^{\infty} d \omega \int_{0}^{\infty} d q q^{3} e^{-2 q d} \\
& \times\left\{\frac{\operatorname{Im} R_{1 p}(\omega) \operatorname{Im} R_{2 p}(\omega)}{\left|1-e^{-2 q d} R_{1 p}(\omega) R_{2 p}(\omega)\right|^{2}}\left(-\frac{\partial n(\omega)}{\partial \omega}\right)+[p \rightarrow s]\right\},
\end{aligned}
$$

For $d<d_{W} \varepsilon^{-1 / 2}$, where $\varepsilon(\omega)$ is taken at the characteristic frequency $\sim k_{B} T / \hbar$, the $p$-wave contribution is given by ${ }^{7}$

$$
\sigma_{p} \approx 0.3\left(k_{B} T / \hbar \omega_{p}\right)^{2} \frac{1}{\left(\omega_{p} \tau\right)^{2}} \frac{\hbar V}{d^{4}},
$$

and for $d_{W} \varepsilon^{-1 / 2}<d<d_{W}$ :

$$
\sigma_{p} \approx \frac{9}{2 \pi^{2}} \frac{\hbar V}{d^{2} d_{W}^{2}} \frac{k_{B} T}{\hbar \omega_{p}} \frac{1}{\omega_{p} \tau} .
$$

For $d<d_{W} \varepsilon^{-1 / 2}$, the $s$-wave contribution $\sigma_{s}$ becomes independent of $d$ :

$$
\sigma_{s} \approx 0.5 \pi^{-2} \hbar^{-1} c^{-4} k_{B}^{2} T^{2} \tau^{2} \omega_{p}^{4} V
$$

For $d>d_{W} \varepsilon^{-1 / 2}$ we have

$$
\sigma_{s} \approx 3.88 \pi^{-2} d^{-2} c^{-2} k_{B}^{2} T \tau \omega_{p}^{2} V
$$

From the comparison (65) and (67) we find that $\sigma_{s}>\sigma_{p}$ for $d>c / \omega_{p}^{2} \tau$. For typical metals at room temperature this corresponds to $d>1 \AA$. This is in drastic contrast to the (conservative) van der Waals interaction, where the retardation effects become important only for $d>c / \omega_{p} \cdot{ }^{6}$ Finally, we carry out the transition to frictional stress between a particle with the radius $R \ll d$ and semi-infinite body in Eq. (64). To do this, we assume as in Sec. IV that the body $\mathbf{2}$ is sufficiently rarefied, i.e., that the difference $\varepsilon_{2}-1$ is small. Keeping only the first nonvanishing terms in the expansion of the integrand of Eq. (64) in powers of these difference, we get a formula similar to Eq. (28):

$$
\begin{aligned}
\sigma= & \frac{\hbar V}{\pi} \int_{0}^{\infty} d \omega\left(-\frac{\partial n(\omega)}{\partial \omega}\right) \int_{0}^{\infty} d q q^{4} e^{-2 q d} \\
& \times\left\{2 \operatorname{Im} R_{1 p}(\omega) \operatorname{Im} \alpha_{2}(\omega)\right. \\
& \left.+\left(\frac{\omega}{c q}\right)^{2} \operatorname{Im} R_{1 s}(\omega) \operatorname{Im} \alpha_{2}(\omega)\right\} .
\end{aligned}
$$

In the limit $d<|\varepsilon|^{-1 / 2} d_{W}$ the reflection factor of the $p$ wave is determined by Eq. (29) and in Eq. (69) the $p$-wave contribution is reduced to the formula that was obtained by Tomassone and Widom. ${ }^{17}$ For the spherical particle in this limit we get

$$
\sigma_{p} \approx \frac{3}{16 \pi^{2}} \frac{\hbar V}{d^{5}}\left(\frac{k_{B} T}{\hbar}\right)^{2} \sigma_{1}^{-1} \sigma_{2}^{-1} R^{3}
$$

and for $|\varepsilon|^{-1 / 2} d_{W}<d<d_{W}$ we get

$$
\sigma_{p} \approx 14 \pi^{-5 / 2} \frac{\hbar V}{d^{4}}\left(\frac{k_{B} T}{\hbar}\right)^{5 / 2} \sigma_{1}^{-1 / 2} \sigma_{2}^{-1} c^{-1} R^{3} .
$$

For $d<|\varepsilon|^{-1 / 2} d_{W}, \sigma_{s}$ is independent of $d$ :

$$
\sigma_{s} \approx 3.3 \times 10^{2} \pi^{-1 / 2} \hbar V \sigma_{1}^{3 / 2} \sigma_{2}^{-1} c^{-5} R^{3}\left(\frac{k_{B} T}{\hbar}\right)^{9 / 2}
$$

and for $|\varepsilon|^{-1 / 2} d_{W}<d<d_{W}$ we get

$$
\sigma_{s} \approx 3.54 \pi^{-3 / 2} \frac{\hbar V}{d^{4}}\left(\frac{k_{B} T}{\hbar}\right)^{5 / 2} \sigma_{1}^{-1 / 2} \sigma_{2}^{-1} c^{-1} R^{3} .
$$

From the comparison (70) and (72) we get that $\sigma_{s}>\sigma_{p}$ for $0.1\left(c d_{W} / \sigma_{1}\right)^{1 / 2}<d<|\varepsilon|^{-1 / 2} d_{W}$. For a normal metal at room temperature this corresponds to $10^{2}<d<10^{3} \AA$. For $|\varepsilon|^{-1 / 2} d_{W}<d<d_{W}, \sigma_{s} \approx \sigma_{p}$.

To estimate $\sigma$ for $R \gg d$, we use the same approach as in Sec. IV. We define the frictional stress between the elementary volume $d V$ and the semi-infinite body as $d \sigma$ $=\left(3 \sigma / 4 \pi R^{3}\right) d V$, where $\sigma$ is given by an expression for a spherical particle for $R \ll d$. After integration over the volume of the sphere for $d \ll R<|\varepsilon|^{-1 / 2} d_{W}$ we get 


$$
\sigma_{p} \approx 4 \cdot 10^{-5} \frac{\hbar V}{d^{3}}\left(\frac{k_{B} T}{\hbar}\right)^{2} \sigma_{1}^{-1} \sigma_{2}^{-1} R
$$

Because in this limit $\sigma_{s}$ does not depend on $d$, it is still determined by Eq. (73). From Eqs. (73) and (74) we get that $\sigma_{s}>\sigma_{p}$ for

$$
d>2.4 \cdot 10^{4}\left(c d_{W} \sigma_{1}^{-1}\right)^{5 / 6} R^{-2 / 3} .
$$

For a "normal" metal at room temperature and $R \sim 10^{3} \AA$, $d>10 \AA$.

Recently, Dorofeev et al. ${ }^{15}$ have observed Brownian motion of a small metal particle connected by a spring to a holder, and located in ultrahigh vacuum in the vicinity of a gold surface. It was observed that the particle performed a stochastic oscillatory motion increased as the particle approaches the gold surface. It was suggested that this increased damping is due to the coupling to the fluctuating electromagnetic field. From Eqs. (72) and (74) we can estimate the damping constant $\gamma / m=\sigma / m V$. For $d \sim 10^{2} \AA$, $R \sim 10^{3} \AA, m \sim 10^{-11}-10^{-13} \mathrm{~kg}$, and for "normal" metal at room temperature we get $\gamma_{p} / m \sim 10^{-17} \mathrm{~s}^{-1}$ and $\gamma_{s} / m$ $\sim 10^{-13} \mathrm{~s}^{-1}$. However in the experiment ${ }^{15} \gamma / \mathrm{m} \sim 10^{2} \mathrm{~s}^{-1}$. Thus, the contribution of a fluctuating electromagnetic field to the damping constant cannot explain the observed experimental date. This result is in agreement with our earlier conclusion. $^{14}$

The fluctuating electromagnetic field is an origin of the frictional drag observed between parallel two-dimensional electron systems. ${ }^{18}$ In the frictional drag experiments a current is drawn in the first layer, while the second layer is an open circuit. Thus, no dc current can flow in the second layer, but an induced electric field occurs that opposes the "drag force" from the first layer. Recently, we used the theory of a fluctuating electromagnetic field to calculate the frictional drag force between two-dimensional electron systems. ${ }^{19}$ For frictional drag stress we found a formula that is very similar to Eq. (64). We found that for modulationdoped semiconductor quantum wells, retardation effects are not important under typical experimental conditions, supporting earlier calculations where retardation effects always have been neglected. ${ }^{18,20}$ A striking result we found is that for systems with high two-dimensional electron density, e.g., thin metallic films, retardation effects become crucial and in fact, dominate the frictional shear stress $\sigma$.

\section{SUMMARY AND CONCLUSION}

We have calculated the heat transfer between (a) two flat surfaces, (b) a spherical particle and a flat surface, and (c) between two spherical particles. For two flat solid surfaces we have presented numerical results for several cases of practical importance, namely for two "normal", (highconductivity) metals (silver), two (high-resistivity) semimetals and two silver metals coated by thin layers $(10 \AA)$ of high-resistivity material. For high-resistivity metals, the p-wave contribution dominates, but for "normal" (highconductivity) metals we found the remarkable result that the $s$-wave contribution dominates even for short separation between the solids. For a spherical particle and a flat surface we have considered both $d \gg R$ and $d \ll R$, where $d$ is the particle-surface separation and $R$ the radius of the particle. For $d \ll R$ we have obtained an exact result in the electrostatic limit, and the approximate formulas that include the retardation effects. We have pointed out the close relationship between the radiative heat transfer between two solids and the vacuum friction ${ }^{7,13}$ that occur when one of the solids slide relative to the other solid. The formalism developed in this paper can be generalized to treat the vacuum friction between bodies with curved surfaces.

\section{ACKNOWLEDGMENTS}

We thank B. Stipe for useful discussions. A.I.V. acknowledges financial support from D.F.G. R.F.B.R. (project No. 01-02-16202), and thanks R. O. Jones for many important references and for help in preparation of the manuscript. We thank J. Harris for useful comments on the text. B.N.J.P. acknowledges B.M.B.F. for a grant related to the GermanIsraeli Project Collaboration "Novel Tribological Strategies from the Nano-to Meso-Scales.",
${ }^{1}$ D. Polder and M. Van Hove, Phys. Rev. B 4, 3303 (1971).

${ }^{2}$ M. L. Levin, V. G. Polevoy, and S. M. Rytov, Zh. Eksp. Teor. Fiz. 792087 (1980) [Sov. Phys. JETP 52, 1054 (1980)].

${ }^{3}$ J. B. Pendry, J. Phys.: Condens. Matter 11, 6621 (1999).

${ }^{4}$ A. Majumdar, Annu. Rev. Mater. Sci. 29, 505 (1999).

${ }^{5}$ S. M. Rytov, Theory of Electrical Fluctuation and Thermal Radiation (Academy of Science of USSR Publishing, Moscow, 1953).

${ }^{6}$ E. M. Lifshitz, Zh. Exsp. Teor. Fiz. 2994 (1955) [Sov. Phys. JETP 2, 73 (1956)].

${ }^{7}$ A. I. Volokitin and B. N. J. Persson, J. Phys.: Condens. Matter 11, 345 (1999); Phys. Low-Dim. Struct. 7/8, 17 (1998).

${ }^{8}$ M. L. Levin and S. M. Rytov, Theory of Equilibrium Thermal Fluctuations in Electrodynamics (Science Publishing, Moscow, 1967).
${ }^{9}$ A. A. Abrikosov, L. P. Gor'kov, and I. Ye. Dzyaloshinskii, Quantum Field Theoretical Methods in Statistical Physics (Pergamon, New York, 1965).

${ }^{10}$ B. N. J. Persson and Z. Zhang, Phys. Rev. B 57, 7327 (1998).

${ }^{11}$ L. D. Landau and E. M. Lifshitz, Electrodynamics of Continuous Media (Pergamon, New York, 1975).

${ }^{12}$ R. Nozawa, J. Math. Phys. 7, 1841 (1966).

${ }^{13}$ J. B. Pendry, J. Phys.: Condens. Matter 9, 10301 (1997).

${ }^{14}$ B. N. J. Persson and A. I. Volokitin, Phys. Rev. Lett. 84, 3504 (2000).

${ }^{15}$ I. Dorofeev, H. Fuchs, G. Wenning, and B. Gotsman, Phys. Rev. Lett. 83, 2402 (1999).

${ }^{16}$ G. V. Dedkov and A. A. Kyasov, Phys. Lett. A 259, 38 (1999).

${ }^{17}$ M. S. Tomassone and A. Widom, Phys. Rev. B 56, 4938 (1997).

${ }^{18}$ T. J. Gramila, J. P. Eisenstein, A. H. Macdonald, L. N. Pfeiffer, 
and K. W. West, Phys. Rev. B 66, 1216 (1991); Surf. Sci. 263, 446 (1992); Phys. Rev. B 47, 12957 (1993); Physica B 197, 442 (1994).

${ }^{19}$ A. I. Volokitin and B. N. J. Persson, J. Phys.: Condens. Matter 14,
859 (2001).

${ }^{20}$ M. B. Pogrebinskii, Fiz. Tekh. Poluprovodn. 11, 637 (1977) [Sov. Phys. Semicond. 11, 372 (1977)]; P. J. Rice, Physica B \& C 117, 750 (1983). 